EPJ Web of Conferences 37, 06009 (2012)

DOI: $10.1051 /$ epjconf/20123706009

(C) Owned by the authors, published by EDP Sciences, 2012

\title{
Meson Production in Antinucleon Annihilation on Nuclei
}

\author{
S. Lourenço ${ }^{1, a}$, H. Lenske ${ }^{1}$, and S. Wycech ${ }^{2}$ \\ 1 Institut für Theoretische Physik, JLU Giessen, Heinrich-Buff Ring 16, D-35392 Giessen \\ 2 National Center for Nuclear Studies, Hoza 69, 00-681, Warsaw, Poland
}

\begin{abstract}
Pion production in coherent antiproton annihilation reactions on nuclei is studied in a fully quantum mechanical description. The elementary elastic $\bar{N} N$ and the $\bar{N} N \rightarrow n \pi$ annihilation vertices are obtained from a $t$-matrix accounting for coupled channels effects. The theoretical amplitudes are used to derive a microscopic $\bar{N} A$ optical model potential and the meson production vertices. Pion nucleus final state interaction are taken into account by adding higher pion-nucleon resonances beyond the $\Delta$-resonance. Nuclear structure aspects are described by HFB-theory. Results for $\bar{p} A, \pi A$ and meson production cross sections are presented.
\end{abstract}

\section{Introduction}

The interactions of antinucleons with nucleons and nuclei are dominated by annihilation processes giving access to a large variety of particle production channels, from pions over heavier mesons to baryon-antibaryon production. The underlying annihilation processes are still not completely understood and ask therefore for a consistent theoretical study. In our work [1], we aim for a fully quantum mechanical description of the interaction of antinucleons with nucleons and nuclei. At present we are focusing on investigations of two-meson production on nuclei. This includes the initial state interactions discussed in the following section 2 , the final state interactions of the emitted mesons with the residual nucleus, presented in section 3 and the particle production in the reaction stage which will be discussed in section 4 . The results are of interest for a large variety of investigations with antiprotons planned for the upcoming FAIR@GSI facility.

\section{Initial State Interaction}

The interaction of antinucleons with nuclei is dominated by annihilation processes, finally producing a broad distribution of pions centered around a mean-value of 5 [2]. Here, we are interested in reactions at incident kinetic energies of a few $\mathrm{MeV}$ up to a few GeV. In this energy domain, $\bar{N} A$ interactions are reliably well described by a hadronic picture. The elementary $\bar{N} N$ reaction dynamics include a purely elastic part, obtained by the corresponding G-parity transformed $N N$ interaction, and a dispersive part leading to strong absorption. In the purely hadronic picture underlying our work the dispersive processes are given by intermediate meson production through $t / u$-channel baryon exchange and $s$-channel resonance formation which recombine into the $\bar{N} N$ system. As far as elastic scattering is concerned, the subtleties of $\bar{N} N$ and $\bar{N} A$ interactions can be accounted for by optical potentials. Approaches to free space $\bar{N} N$ interactions like the Paris [3] and the Juelich [4] model are based on the optical model concept. Applications to $\bar{N} A$ scattering are discussed in depth e.g. in [5,6], and they are also used in a microscopic formulation for nuclear structure studies as in our recent work [7].

As in ref. [7] we describe the initial state $\bar{N} A$ interactions in a microscopic model. Since the dominant

\footnotetext{
a e-mail: stefanie.lourenco@physik.uni-giessen.de
} 
part of the annihilation process is already present in free-space $\bar{N} N$ interactions we consider a leading order approach to $\bar{N} A$ interactions. Using the $t-\rho$ approximation the elastic antinucleon-nucleon $(\bar{N} N) t$-matrix is folded with self-consistently calculated Hartree-Fock-Bogoliubov (HFB) densities $\rho_{N}$. Carrying out the calculations in momentum space, we find

$$
U_{o p t}(\mathbf{r})=V(\mathbf{r})-i W(\mathbf{r})=\sum_{N=p, n} \int \frac{d^{3} q}{(2 \pi)^{3}} \rho_{N}(q) t_{\bar{p} N}\left(T_{L a b}, q^{2}\right) e^{i \mathbf{q} \cdot \mathbf{r}}
$$

The $\bar{N} N$ amplitudes are taken either from microscopical models such as Juelich-Bonn model [4] and Paris model [3] or, at energies beyond the range of validity of the models, they are derived by means of the optical theorem from the $\bar{N} N$ total cross sections. In the Juelich-Bonn [4] model the amplitudes are constructed by explicitly calculating two-meson intermediate states, while all other contributions are subsumed into an $\bar{N} N$ an optical potential. As will be discussed in sect. 4, the same type of diagrams are entering also into the description of two-meson production where they are evaluated on the energy shell while here they are contributing as virtual intermediate channels. At this instance, we neglect special many-body effects, e.g. the multi-nucleon annihilation mechanism [8]. While such effects are not showing up as prominent features in the global annihilation process, they will affect significantly reaction channels like the Pontecorvo single pion production process [9].

As discussed in some detail in [7], in practice the $\bar{N} A$ scattering wave functions and cross sections are typically calculated in eikonal approximation, except for lowest energies where a fully quantum mechanical approach is used by solving numerically the Schrödinger equation in partial wave representation. Results can be found in ref. [7].

\section{Final State Interaction}

Despite the fact that meson-nucleus interactions have been studied extensively in the past there is surprisingly few known about interactions in asymmetric nuclear matter. This and the fact that there is a lack of knowledge on pion-nucleus interactions above $T_{L a b} \sim 1 \mathrm{GeV}$ indicates the need for reconsidering pion self-energies in nuclear matter. Low-energy studies around the prominent $\pi+N \leftrightarrow \Delta_{33}(1232)$ resonance show that basically the $\pi A$ optical potentials contain isoscalar and isovector contributions, following closely the elementary $\pi N s$-wave and $p$-wave processes. Microscopically, they are understood by $t$-channel meson-exchange, formation of resonances in the $\pi N$ system. In nuclear matter, they are supplemented by genuine many-body contributions from particle - hole excitations of the nuclear medium and higher order multi-nucleon processes [10,11]. Because in our case the mesons are produced in an $\bar{N} N$ annihilation process their self-energies need to be described over a large energy range. We use a Kisslinger-type potential $U_{\pi A}=U_{s}+\vec{\partial} U_{p} \vec{\partial}$ including the aforementioned $s$-wave $\left(U_{s}\right)$ and $p$-wave $\left(U_{p}\right)$ components, respectively, and to be used in a Klein-Gordon equation. By a Krell-Ericson transformation for the wave function, $\Phi=\left(1-U_{p}\right)^{-1 / 2} \psi$, we obtain the equivalent local momentum-dependent potential

$$
U_{\text {local }}(r)=\frac{(\hbar c)^{2}}{2 \omega}\left\{\frac{U_{s}}{1-U_{p}}-\frac{k^{2} U_{p}}{1-U_{p}}-\left[\frac{\frac{1}{2} \nabla^{2} U_{p}}{1-U_{p}}+\left(\frac{\frac{1}{2} \nabla U_{p}}{1-U_{p}}\right)^{2}\right]\right\} .
$$

As the two mesons are produced in antinucleon-nucleon annihilation, already at rest an energy of about $\sqrt{s} \sim 2 \mathrm{GeV}$ is available and will be shared by the emerging mesons. The pion-nucleon interaction is dominated by the intermediate formation of $N^{*}$ resonances, where at low energies the $\Delta(1233)$ resonance is the most prominent one, but with increasing energy also other resonant states become accessible. Hence, we have to extend existing approaches as [12] by adding to the optical potential higher 4-star and 3-star PDG-resonances [13] as intermediate states in the energy region of interest. We obtain a good description not only for a large energy range, but also for different nuclei. As an example, the angle integrated total cross section (in eikonal approximation) of ${ }^{6} \mathrm{Li}$ is shown in Fig. 1. 

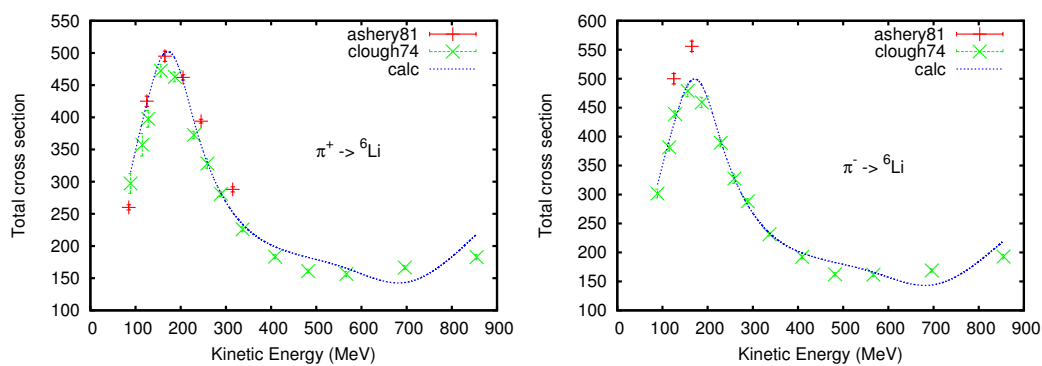

Fig. 1. Total cross section plotted against the laboratory momentum. Eikonal results obtained with the extended Kisslinger-type optical potential (dashed line) are compared to data (crosses). Both, $\pi^{+}$and $\pi^{-}$can be well described in the interested energy range. The extension to higher energies is done by adding higher resonances of Breit-Wigner-type. The data are taken from [14] and [15], respectively.

\section{Meson Production by Antinucleon Annihilation on Nuclei}

Coherent meson production by antinucleon annihilation on nuclei can proceed, in principle, by two reaction mechanisms, namely by pickup or in-flight annihilation and by in situ annihilation. The pickup annihilation process is a sequential process where first a nucleon is stripped off the target and forms a short-lived intermediate $\bar{N} N$ state which then annihilates into hadrons. From a nuclear structure point of view this corresponds to a stripping reaction leaving the residual nucleus in a well defined quantum state. The competing in situ annihilation mechanism, on the other hand, amounts to stop the antinucleon in the target matter, followed by annihilation into hadrons. In this case, rescattering and absorption processes of the produced hadrons will be of particular importance. A common feature of both production mechanisms is the difference of scales: Nuclear dynamics is governed by momenta of the order of the Fermi momentum, $k_{F} \sim 250 \mathrm{MeV} / \mathrm{c}$. The elementary production is determined by baryon exchange, involving momenta of at least $q \sim 1 \mathrm{GeV} / \mathrm{c}$ corresponding to extremely short-ranged interactions of almost point-like character.

Here, we concentrate on two-meson production, described by the cross section:

$$
d^{9} \sigma_{\alpha \beta}=N_{\alpha \beta}\left(\frac{\hbar c}{2 \pi}\right)^{9} \frac{d^{3} k_{1}}{E_{1}} \frac{d^{3} k_{2}}{E_{2}} \frac{d^{3} k_{B}}{E_{B}}\left|M_{\alpha \beta}\left(\mathbf{k}_{\mathbf{1}}, \mathbf{k}_{\mathbf{2}}, \mathbf{k}_{\mathbf{B}} ; \mathbf{k}_{\alpha}\right)\right|^{2} \delta\left(\mathbf{k}_{\mathbf{1}}+\mathbf{k}_{\mathbf{2}}+\mathbf{k}_{\mathbf{B}}\right) \delta\left(E_{1}+E_{2}+E_{B}-\sqrt{s}\right)
$$

where the $\delta$-distribution accounts for the energy and momentum conservation. Altogether the interesting physics of particle production is contained in the matrix element:

$$
M_{\alpha \beta} \approx t_{\bar{N} N \rightarrow 2 \pi}(s)\left\langle\chi_{1 \beta}^{(-)} \chi_{2 \beta}^{(-)}\left|\varphi_{B}\right| \chi_{\bar{N} A}^{(+)}\right\rangle
$$

The scale differences allow to decouple to a large extent the elementary production process and the nuclear structure parts. The essential features of the reaction process are already described by a contact interaction with a energy dependent strength, as indicated above. The nuclear properties are taken into account by the overlap wave function $\varphi_{B}$, obtained from HFB-calculations. Initial and final state interactions are contained in the corresponding distorted waves $\left(\chi_{1 \beta}^{(-)} \chi_{2 \beta}^{(-)} \chi_{\bar{N} A}^{(+)}\right.$, respectively). As a first example we consider production in the $\rho$-meson channel, integrating out relative motion of the final pions. Results for the total cross section, obtained in eikonal approximation, are shown in Fig. 2.

\section{Summary}

Meson production by coherent antinucleon annihilation on nuclei is investigated microscopically by taking into account initial and final state interactions and describing the production process in a hadronic model. Nuclear structure contributions are incorporated by state-of-the art HFB calculations. 


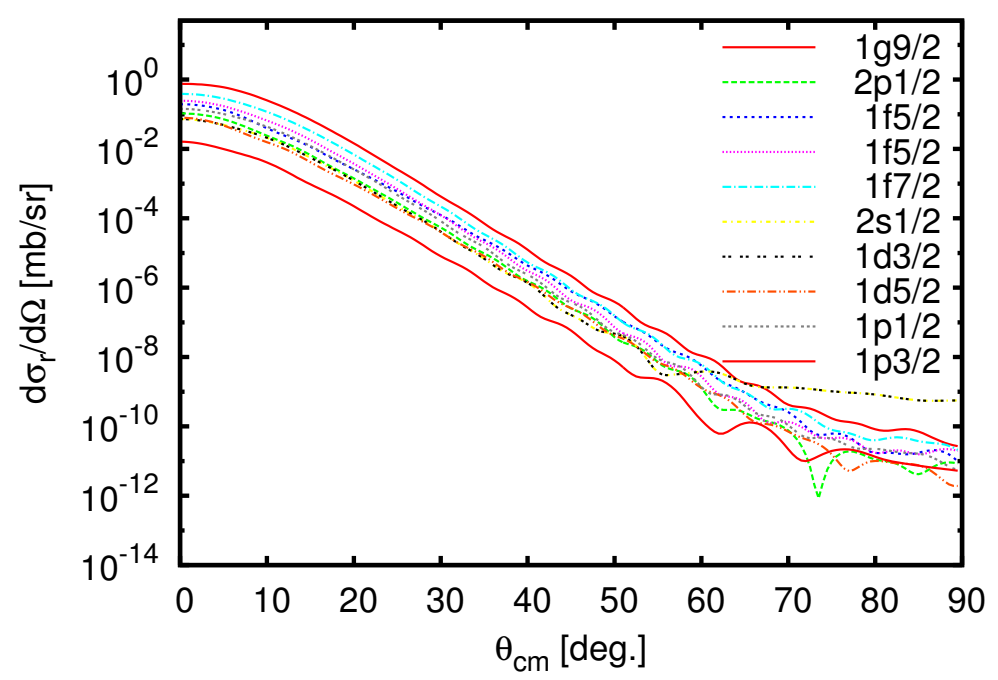

Fig. 2. Total cross section for meson production in the $\rho^{0,-}$ channel decaying into two pions. The differential cross sections are shown for meson production on a ${ }^{78} \mathrm{Ni}$ target and $T_{l a b}=800 \mathrm{MeV}$.

The pickup and the in situ reaction mechanisms as competing production processes were introduced. First results for meson production in antiproton-annihilation on nuclei have been presented. It was pointed out that the final state interactions of two produced pions require to extend existing approaches by including higher resonances. A good description of the total pion nucleus interaction for various energies and nuclei could be presented. Work is in progress to extend the approach to kaon production and Pontecorvo reactions on heavy nuclei without and with strangeness.

\section{References}

1. S. Lourenco et al., Hyp. Int. 209, (2012) 117.

2. M. R. Clover et al., Phys. Rev. C 26, (1982) 2138.

3. B. El-Bennich et al., Phys. Rev. C 79, (2009) 054001.

4. V. Mull et al., Phys. Rev. C 51, (1995) 2360.

5. A.M. Green, S. Wycech, Nucl. Phys. A 377, (1982) 441.

6. C.J. Batty, E. Friedman, A. Gal, Phys. Rep. 287, (1997) 385.

7. H. Lenske, P. Kienle, Phys. Lett. B 647, (2007) 82.

8. E. Hernandez, E. Oset, Z. Phys. A 342, (1992) 201.

9. B.H. Pontecorvo, Zh. Eksp. Teor. Fiz. 3, (1956) 1143; Sov. Phys. JETP 30, (1956) 947.

10. N. Kaiser, W. Weise, Phys. Lett. B 512, (2001) 283.

11. M. Doering, E. Oset, Phys. Rev. C 77, (2008) 024602.

12. M. B. Johnson et al., Ann. Phys. 248, (1996) 134.

13. J. Beringer et al. (Particle Data Group), Phys. Rev. D 86, (2012) 010001.

14. D. Ashery et al., Phys. Rev. C 23, (1981) 2173.

15. A. S. Clough et al., Nucl. Phys. B 76, (1974) 15. 